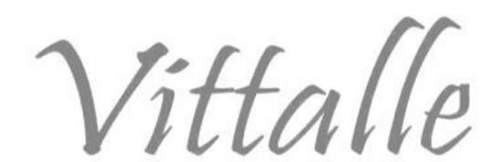

\title{
Perfil clínico, epidemiológico e microbiológico dos pacientes internados no setor de cirurgia geral do Hospital Universitário da Universidade Federal do Vale do São Francisco \\ Yasmin Bione Diniz Amando ${ }^{a^{*}}$, Bruno Saraiva Bezerra Medrado ${ }^{a}$, Sandra Lúcia Lodi Peres $^{b}$, Hugo Alessi Soares ${ }^{\mathrm{a}}$, Carine Rosa Naue ${ }^{\mathrm{b}}$
}

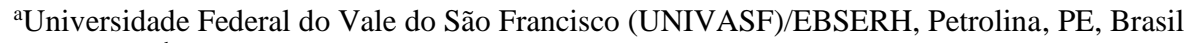

${ }^{b}$ Hospital Universitário da UNIVASF/EBSERH, Petrolina, PE, Brasil

Histórico do Artigo:

Recebido em

23/04/2020

Aceito em

07/08/2020

\section{Palavras-chave:}

Infecção hospitalar; cirurgia abdominal; cirurgia torácica; bactérias; resistência; sensibilidade

\section{Keywords:}

Nosocomial Infection; abdominal surgery; thoracic surgery; bacteria; resistance; sensitivity

\begin{abstract}
RESUMO
Infecção Hospitalar é aquela adquirida após a admissão do paciente, cuja manifestação ocorreu durante a internação ou após a alta e é considerada um problema de saúde pública. Este trabalho tem como objetivo analisar o perfil clínico, epidemiológico e microbiológico de pacientes internados no setor de Cirurgia Geral no Hospital Universitário da UNIVASF, no período de março a setembro de 2018. Foi realizado um estudo retrospectivo com abordagem quantitativa, tendo como fonte de informação prontuários eletrônicos e dados disponibilizados pelo Aplicativo de Gestão para Hospitais Universitários (AGHU). As variáveis analisadas para o perfil clínico e epidemiológico foram idade, sexo, procedência, motivo de internação, período de internação, quantidade de dias de internação, necessidade de internação em Unidade de Terapia Intensiva (UTI) e a quantidade de dias que permaneceu nesta unidade, uso de antimicrobiano prévio,número de cirurgias realizadas e foco principal da infecção.Já o perfil microbiológico teve como base todas as culturas positivas. Os dados foram tabulados no aplicativo GOOGLE FORMS e sintetizados em gráficos e tabelas para análise descritiva dos dados. Foram selecionados para participar do estudo 66 pacientes com culturas positivas. Separou-se quanto ao foco da infecção, $45,5 \%$ foram de origem abdominal, $34,8 \%$ torácicas/pulmonares, $12,1 \%$ de partes moles, $4,5 \%$ mistas e 3\% de infecção de ferida operatória. Dentre as bactérias mais encontradas se destacaram Acinetobacter baumannii, Escherichia coli, e Klebsiella sp. Em relação ao perfil dos isolados, no geral, pode-se observar uma elevada resistência aos antibióticos analisados. Pode-se concluir que a maioria dos pacientes foram do sexo masculino, provenientes da emergência e as infecções estão mais relacionadas ao abdômen e ao tórax.
\end{abstract}

Clinical, epidemiological and microbiological profile of patients hospitalized in the general surgery sector of the University Hospital of the Federal University of the Valley of São Francisco

\section{ABSTRACT}

Hospital-acquired infection (HAI), also known as nosocomial infection, is the one acquired during or after hospital admission, which symptoms and signs occurred during hospitalization period or after discharge and it is considered a public health problem. This article has the purpose to analyses the clinical, epidemiologic, and microbiologic profile of hospitalized patients in general surgery sector of University Hospital of UNIVASF, in the period of March to September in 2018. The retrospective study was designed in a quantitative approach, having as data source the electronic medical records availed in the AGHU system. The studied variables for clinical and epidemiologic profile were age, sex, precedence, hospitalization causes, need of intensive therapy, hospitalization time (in days), previous antibiotic therapy, number of surgeries performed and main infection site. The microbiologic profile was designed based on all positive bacterial culture. The data was organized on GOOGLE FORMS Application and synthesized in graphics and tables for descriptive analyses. Were selected to be part of the study 66 patients with positive bacterial culture. It was separated as to infection sites, where $45,5 \%$ were from abdominal site, $34,8 \%$ thoracic/pulmonary, $12,1 \%$ from soft tissues, $4,5 \%$ mixed site, and $3 \%$ from surgical wound. Among the most found bacteria, special feature for Acinetobacter baumannii, Escherichia coli, and Klebsiella sp. Regarding the profile of the isolates, in general, a high resistance to the antibiotics analyzed can be observed. It can be concluded that most patients were male, from the emergency room and the infections are more related to the abdomen and thorax.

\footnotetext{
* Autor correspondente: yasminbionediniz@gmail.com (Amado Y.B.D.)
} 


\section{Introdução}

O traumatismo abdominal é responsável por um número expressivo de mortes evitáveis. As complicações pós-operatórias comuns incluem complicações sistêmicas como pneumonia, sepse e disfunção renal e tromboembolismo e complicações específicas da cirurgia abdominal, como infecção do local cirúrgico, abscesso intraabdominal e sepse abdominal $(1,2)$.

A pneumonia e o empiema são complicações decorrentes de drenagem torácica em 1 a $3 \%$ dos casos. Quanto maior o tempo de permanência do dreno e do hemotórax retido maior o risco de infecção (3).

O Ministério da Saúde define Infecção Hospitalar (IH) como aquela adquirida após a admissão do paciente cuja manifestação ocorreu durante a internação ou após a alta, podendo ser relacionada com a internação ou com procedimentos hospitalares (4).

As infecções mais comuns em ambientes hospitalares, são as pneumonias associadas a ventilação, infecções do trato urinário associadas a cateter, infecções da corrente sanguínea associada a acesso central, infecções de feridas cirúrgicas e do trato gastrintestinal (5).

Staphylococcus aureus e Staphylococcus epidermidis são organismos comuns que causam ISC entre pacientes submetidos a procedimentos torácicos. Patógenos isolados em pacientes com pneumonia pós-operatória são diversos e incluem organismos Grampositivos (Streptococcus e Staphylococcus), gram-negativos (Haemophilus influenzae, Enterobacter cloacae, Klebsiella pneumoniae, Acinetobacter sp., Pseudomonas aeruginosa e a Moraxella catarrhalis) e fungos (Candida sp.) (6).

O risco de transmissão de patógenos é variável e depende do status imunológico do paciente, da prevalência local de vários patógenos e das práticas de controle de infecção e administração antimicrobiana utilizadas durante a hospitalização (7). Além disso, a resistência microbiana vem sendo considerada uns dos maiores desafios aos sistemas de saúde contemporâneos (8).

Entre as bactérias resistentes causadoras de infecção em hospitais, destacam-se como as mais frequentes: Staphylococcus aureus resistentes à meticilina (MRSA), Enterococcus resistentes à vancomicina (VRE) e Staphylococcus coagulase-negativa (SCN) com perfil de sensibilidade reduzida e resistência à oxacilina e a cefalotina, podendo apresentar resistência cruzada a $\beta$-lactâmicos e outras classes de antibióticos. $(9,10,11)$. Entre os bacilos gram negativos, ganham destaque: Escherichia coli e Klebsiella pneumoniae produtoras de B-lactamase de espectro estendido (ESBL) incluindo a produção de carbapenemase, E. coli resistentes a fluorquinolonas, Pseudomonas aeruginosa e Acinetobacter baumanni resistentes aos carbapenêmicos e Enterobacter sp. resistentes a aminoglicosídeos, cefalosporinas de $3^{\text {a }}$ geração e fluoroquinolonas $(12,13)$.

Diante da importância dos quadros de infecções hospitalares para especialidade de Cirurgia Geral e a necessidade do conhecimento dos perfis destes pacientes para tomada de decisões, este trabalho tem como objetivo analisar o perfil clínico, epidemiológico e microbiológico dos pacientes internados no setor de cirurgia geral no Hospital Universitário da Universidade Federal do Vale do São Francisco (Petrolina-PE).

\section{Matériais e métodos}

Trata-se de um estudo retrospectivo, descritivo e documental com abordagem quantitativa, realizado durante o período de março a setembro de 2018, em um Hospital 
Universitário no município de Petrolina-PE.

A coleta de dados clínicos, epidemiológicos e microbiológicos ocorreu a partir de informações contidas em prontuários eletrônicos, através do Aplicativo de Gestão para Hospitais Universitários (AGHU) da unidade. A pesquisa teve aprovação do Comitê de ética e pesquisa tendo como número de CAAE: 66493917.0.0000.5196.

Foram incluídos prontuários de todos os pacientes internados no setor de cirurgia geral que possuíam cultura positiva durante o período de março a setembro de 2018. Foram excluídos os pacientes de outras áreas clínicas ou cirúrgicas que não sejam da cirurgia geral e pacientes que ficaram internados por mais de dois meses no hospital. Estes foram excluídos devido aos altos índices de colonização de múltiplos microorganismos, prejudicando os resultados das culturas e modificando o foco da pesquisa.

O perfil clínico e epidemiológico teve como variáveis: idade, sexo, procedência, motivo de internação, período da hospitalização, quantidade de dias de internamento, necessidade de tratamento em uti e a quantidade de dias que permaneceu nesta unidade, uso de antimicrobiano prévio, número de cirurgias realizadas e foco principal da infecção. Já o perfil microbiológico teve como base as hemoculturas, culturas de secreções traqueais, uroculturas, culturas de líquidos, culturas de partes moles e culturas de ferida operatória dos pacientes envolvidos na pesquisa, tendo como variáveis os micro-organismos isolados, sensibilidade e resistência antimicrobiana. As identificações das bactérias e os antibiogramas foram executados através do sistema automatizado PHOENIX, da BD, utilizando-se os painéis adequados.

Os dados disponibilizados pelo AGHU foram coletados e digitados em arquivos através de uma ficha padronizada na plataforma de dados GOOGLE FORMS. Através desta, foram confeccionadas planilhas e gráficos e realizada análise descritiva com valores absolutos e em percentuais, sendo realizada predominantemente análise descritiva, em que se determinou a frequência das variáveis estudadas, bem como médias.

\section{Resultados}

Durante o período do estudo, foram internados aos cuidados da equipe de cirurgia geral do hospital, 473 pacientes, destes foram selecionados 66 pacientes, que correspondem aos pacientes com culturas positivas. Dos 407 excluídos, 402 não apresentavam culturas positivas e 5 permaneceram internados por mais de 60 dias.

A maioria dos pacientes selecionados pertencia ao gênero masculino $(\mathrm{N}=48 ; 72,7 \%)$, enquanto apenas $18(27,3 \%)$ eram do sexo feminino. Os pacientes foram separados de acordo com a procedência, $52(78,8 \%)$ foram provenientes da emergência e $14(21,2 \%)$ encaminhados do ambulatório.

A idade dos pacientes variou de 16 a 97 anos, tendo uma média de 44,3 anos. No entanto, a idade variou de acordo com a procedência, dentre os pacientes encaminhados do ambulatório a média de idade foi de 61 anos, enquanto a média da idade daqueles provenientes da emergência foi de 39,8 anos.

Os indivíduos incluídos na pesquisa tiveram uma permanência hospitalar variando entre 1 e 43 dias, com uma média de 15,1 dias. Dentre aqueles provenientes da emergência, a média foi 16,2 dias, enquanto os encaminhados do ambulatório a média foi 11 dias, evidenciando que por mais que os pacientes provenientes do ambulatório sejam mais idosos e com mais comorbidades associadas, os pacientes advindos da emergência acabam ficando mais tempo internados.

Quanto ao motivo de internação, 11 pacientes deram entrada no hospital devido abdome agudo inflamatório, 9 por infecção de partes moles, 7 por perfurações por arma 
de fogo, 6 devido a perfurações por arma branca, 7 por trauma torácico fechado, 6 por neoplasias de vias biliares ou pancreáticas, 5 por politraumatismos, 4 por neoplasia do trato gastrointestinal, 3 por abdome agudo perfurativo, 3 por trauma abdominal fechado, 2 para realização de reconstrução do trânsito intestinal, 1 por infecção do trato respiratório, 1 por abdome agudo vascular e 1 por abdome agudo obstrutivo.

A maior parte dos pacientes não necessitou de internação em Unidade de Terapia Intensiva (UTI), apenas 19,7\% permaneceram pelo menos um dia em UTI. Destes, $30,8 \%$ eram provenientes do ambulatório, enquanto 69,2\% vieram da emergência. Em relação ao tempo de permanência nas unidades os pacientes da emergência ficaram em média 14,7 dias enquanto os do ambulatório ficaram apenas 1,7 dias.

Dos pacientes incluídos no estudo, 89,4\% realizaram algum tipo de intervenção cirúrgica. Além disso, quanto ao número de cirurgias realizadas, 59,3\% realizaram 1 cirurgia, 32,2\% realizaram 2 cirurgias, 3,4\% realizaram 3 cirurgias, 1,7\% realizaram 4 cirurgias e $3,4 \%$ realizaram 5 cirurgias.

Quanto ao foco da infecção, $45,5 \%$ eram de origem abdominal, 34,8\% torácicas/pulmonares, $12,1 \%$ de partes moles, $4,5 \%$ mistas (tórax e abdome) e $3 \%$ decorrentes de infecção de ferida operatória.

Quando se refere aos antimicrobianos utilizados durante a internação, ceftriaxona foi utilizada em 39 pacientes, metronidazol em 31, ciprofloxacino em 14, clindamicina em 19, piperacilina-tazobactam em 30, amicacina em 31, meropenem em 10, vancomicina em 7, oxacilina em 6, aciclovir em 1, teicoplanina em 4, levofloxacino em 3, polimixina b em 6, cefalotina em 3, cefepime em 2, tigeciclina em 2, ampicilina-sulbactam em 1, gentamicina em 1, ceftazidima em 1 e linezolida em 1. Além de fluconazol em 9 pacientes e anidulafungina em 2.

Foram analisadas na pesquisa 233 culturas de 66 pacientes, sendo 82 positivas e 149 negativas. Dentre as negativas, as hemoculturas corresponderam a $51,7 \%$, uroculturas a $34,2 \%$, culturas de líquido abdominal $6,7 \%$, líquido torácico $2 \%$, secreção traqueal $3,4 \%$, partes moles $1,3 \%$ e secreção de ferida operatória menos de $1 \%$. Dentre as culturas positivas, $39 \%$ foram culturas de líquido abdominal, $11 \%$ de urocultura, $11 \%$ de partes moles, $11 \%$ de secreção traqueal, $9,8 \%$ de hemoculturas, $9,8 \%$ de líquido torácico, $7,3 \%$ de ferida operatória e $1,2 \%$ de líquor.

No restante das culturas positivas foram isolados 2,4\% de Candida sp.,2,4\% de Moraxella sp.,1,2\% de Gamella morbillorum, 1,2\% de Trueperella pyogenes, 1,2\% de Lactococcus raffinolactis, 1,2\% de Baccilus megaterium, 1,2\% de Shigella boydii, 1,2\% de Shewanella putrefaciens, 1,2\% de Edwardsiella tarda, 1,2\% de Citrobacter freundiie $1,2 \%$ a Aeromonas veronii totalizando uma ocorrência de $15,6 \%$. 


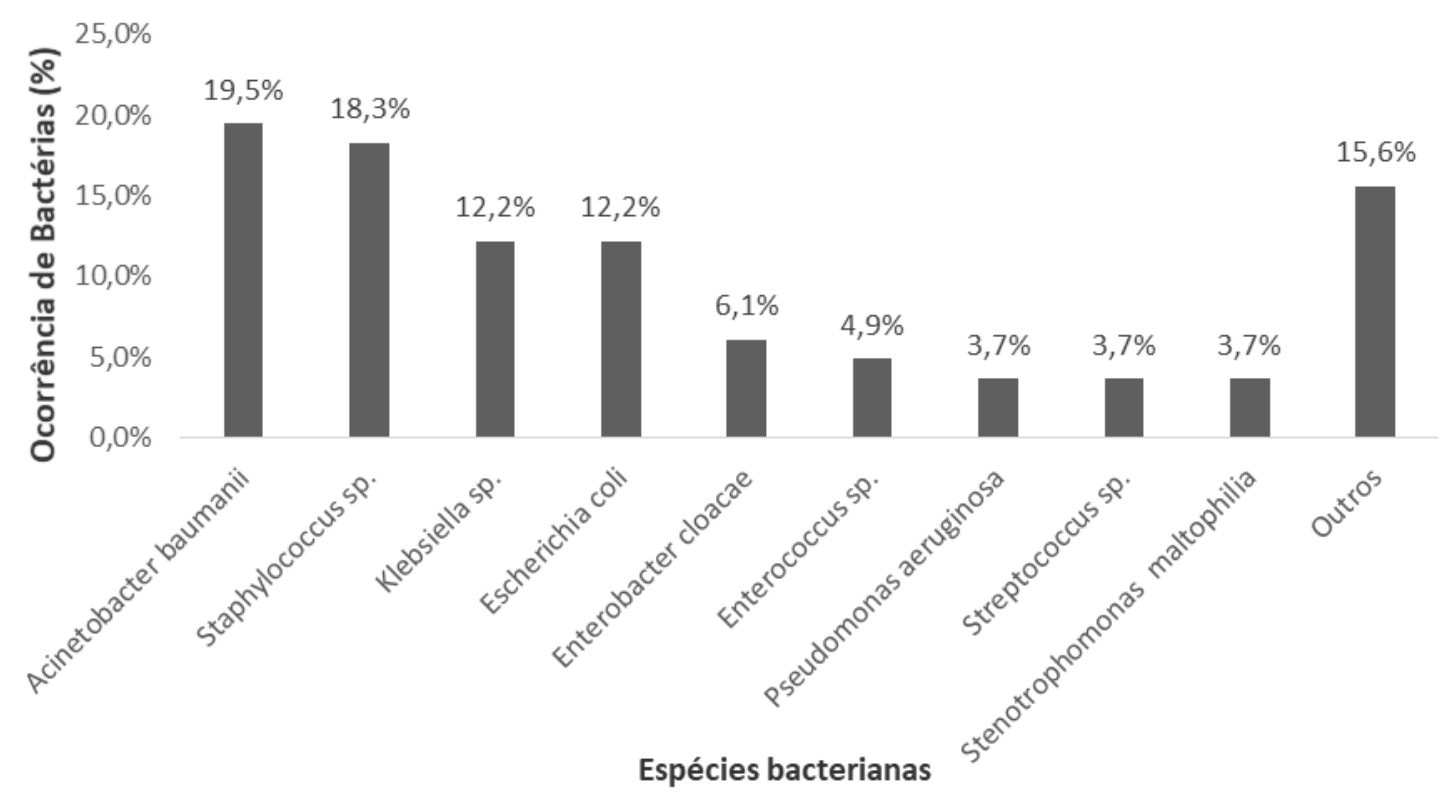

Figura 1 - Ocorrência de bactérias isoladas de amostras biológicas coletadas.

Em relação ao perfil microbiológico das infecções de foco abdominal, foram identificadas 56 culturas positivas quando analisados os prontuários dos pacientes. Dessas, $64,2 \%$ foram de líquido abdominal, $11,3 \%$ relativas a hemoculturas e $24,5 \%$ referentes outros tipos de culturas.

Dos pacientes com infecção de foco abdominal, 17,9\% dos indivíduos tiveram E.coli como bactéria identificada em cultura, 17,9\% A. baumannii, 8,9\% K. pneumoniae, 5,4\% E. cloacae, 5,4\% P. aeruginosa, 5,4\% de S. epidermidis, 5,4\% de $S$. haemolyticus, 3,6\% de S. aureus, $1,8 \%$ de S.lentus, $1,8 \%$ de Enterococcus casseliflavus, $1,8 \%$ de Enterococcus faecium, 1,8\% de Enterococcus hiraee 23,2\% distribuídos entre outros 13 micro-organismos (Figura 2)

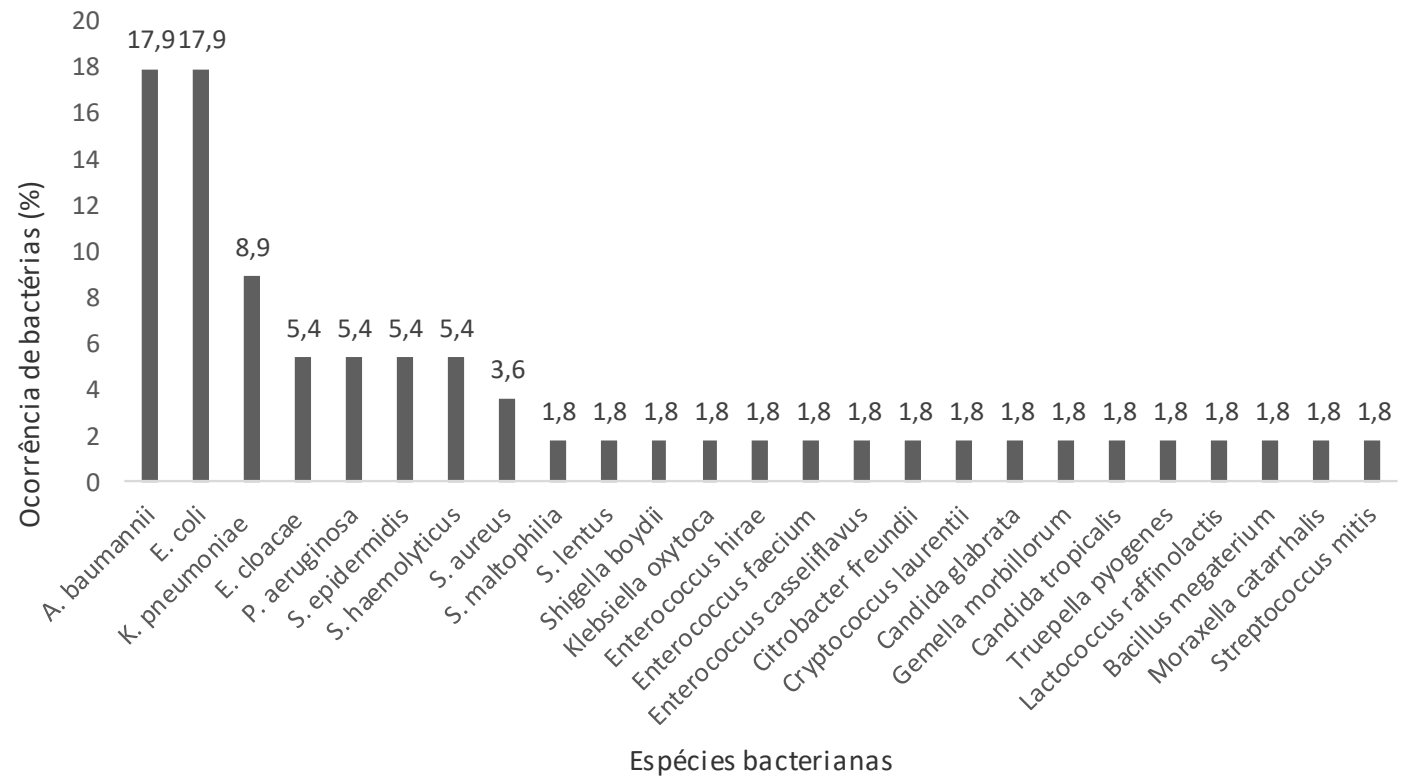

Figura 2 - Ocorrência de bactérias isoladas em culturas coletadas para o diagnóstico de sepse abdominal. 
Quando se refere ao A. baumannii, 90\% foram resistentes a amicacina, cefepime, ceftazidima, ciprofloxacino, gentamicina, imipenem, levofloxacino, meropenem, piperacilina-tazobactam e sulfametoxazol-trimetoprim. O único antimicrobiano que apresentou boa sensibilidade foi a colistina, que se apresentou $100 \%$ sensível. A única exceção fora o ampicilina-sulbactam que apresentou $44 \%$ de resistência e $44 \%$ de intermediário.

No que se refere a E.coli, pode-se observar que $100 \%$ dos isolados foram resistentes à ampicilina-sulbactam, $90 \%$ a ampicilina, $70 \%$ a ceftriaxona, $20 \%$ a gentamicina, $10 \%$ a meropenem e $10 \%$ a imipenem, enquanto $100 \%$ são sensíveis a amicacina, colistina e tigeciclina.

Já em relação as infecções abdominais por K. pneumoniae, $100 \%$ foram resistentes a ampicilina e cefazolina, $80 \%$ a ampicilina-sulbactam, cefepime, ceftriaxona, ciprofloxacino, gentamicina, levofloxacino piperacilina-tazobactam e sulfametoxazoltrimetoprim, $20 \%$ a meropenem. Ao passo que $100 \%$ foram sensíveis a colistina e amicacina. Vale ressaltar que se tratando de tigeciclina e imipenem, $80 \%$ dos casos são sensíveis e $20 \%$ tem sensibilidade intermediária.

Tratando-se de E. cloacae, $100 \%$ dos isolados foram resistentes a ampicilina, ampicilina-sulbactam, cefazolina e cefoxitina. À medida que $100 \%$ foram sensíveis a amicacina, colistina, ertapenem, imipenem, levofloxacino e tigeciclina. Também foi evidenciado que o ciprofloxacino não apresentou resistência a este isolado, sendo $67 \%$ sensível e 33\% intermediário.

Em relação aos pacientes infectados por $P$. aeruginosa, $67 \%$ dos casos foram resistentes a amicacina, cefepime, ceftazidima, ciprofloxacino, gentamicina, imipenem, levofloxacino e meropenem, enquanto100\% dos casos são sensíveis a colistina. Jáa piperacilina-tazobactam, apresentou a mesma porcentagem de resistentes, sensíveis e intermediários, $33,3 \%$ cada.

Sobreo S. epidermidis, $100 \%$ foram resistentes a ampicilina, clindamicina, eritromicina, penicilina $\mathrm{G}$ e rifampicina e $67 \%$ resistentes a oxacilina. Ao mesmo tempo que $100 \%$ apresentaram-se sensíveis a daptomicina e à vancomicina.

Em relação ao S. aureus, $100 \%$ foram resistentes a ampicilina e penicilina G. Enquanto $100 \%$ apresentaram-se sensíveis a ceftarolina, clindamicina, daptomicina, eritromicina, linezolida, minociclina, oxacilina, rifampicina, sulfametoxazoltrimetoprim, tigeciclina e vancomicina (Tabela 1). 
Tabela 1 - Perfil de resistência das bactérias mais ocorrentes isoladas de culturas coletados em pacientes internados no setor de Cirurgia Geral do Hospital Universitário, com diagnóstico de sepse de foco abdominal, no período de março a setembro de 2018

\begin{tabular}{|c|c|c|c|c|c|c|c|c|c|c|c|c|c|c|}
\hline \multirow[t]{3}{*}{ Antimicrobianos } & \multicolumn{8}{|c|}{ Espécies bacterianas } & \multirow{2}{*}{\multicolumn{2}{|c|}{$\begin{array}{c}E . \\
\text { coli }\end{array}$}} & \multirow{2}{*}{\multicolumn{2}{|c|}{$\begin{array}{c}\text { S. } \\
\text { aureus }\end{array}$}} & \multirow{2}{*}{\multicolumn{2}{|c|}{$\begin{array}{c}S . \\
\text { epidermidis }\end{array}$}} \\
\hline & \multicolumn{2}{|c|}{$\begin{array}{c}A . \\
\text { baumannii }\end{array}$} & \multicolumn{2}{|c|}{$\begin{array}{c}P . \\
\text { aeruginosa }\end{array}$} & \multicolumn{2}{|c|}{$\begin{array}{c}K . \\
\text { pneumoniae }\end{array}$} & \multicolumn{2}{|c|}{$\begin{array}{c}E . \\
\text { cloacae }\end{array}$} & & & & & & \\
\hline & $\mathbf{n}$ & $\%$ & $\mathbf{N}$ & $\%$ & $\mathbf{n}$ & $\%$ & n & $\%$ & $\mathbf{n}$ & $\%$ & n & $\%$ & $\mathbf{n}$ & $\%$ \\
\hline Ampicilina & NT & NT & NT & NT & 5 & 100 & 3 & 100 & 10 & 90 & 1 & 100 & 3 & 100 \\
\hline Amicacina & 10 & 90 & 3 & 67 & 5 & 0 & 3 & 0 & 10 & 0 & NT & NT & NT & NT \\
\hline Amp+sub & 10 & 44 & NT & NT & 5 & 80 & 3 & 100 & 8 & 100 & NT & NT & NT & NT \\
\hline Amox +clav & NT & NT & NT & NT & NT & NT & NT & NT & 2 & 50 & NT & NT & NT & NT \\
\hline Cefepime & 10 & 90 & 3 & 67 & 5 & 80 & 3 & 33 & 10 & 60 & NT & NT & NT & NT \\
\hline Ceftarolina & NT & NT & NT & NT & NT & NT & NT & NT & NT & NT & 2 & 0 & NT & NT \\
\hline Ceftazidina & 10 & 90 & 3 & 67 & NT & NT & NT & NT & NT & NT & NT & NT & NT & NT \\
\hline Cefazolina & NT & NT & NT & NT & 3 & 100 & 3 & 100 & 5 & 80 & NT & NT & NT & NT \\
\hline Cefoxitina & NT & NT & NT & NT & 5 & 20 & 3 & 100 & 10 & 20 & NT & NT & NT & NT \\
\hline Cefuroxima & NT & NT & NT & NT & NT & NT & NT & NT & 2 & 50 & NT & NT & NT & NT \\
\hline Ceftriaxona & NT & NT & NT & NT & 5 & 80 & 3 & 33 & 10 & 70 & NT & NT & NT & NT \\
\hline Ciprofloxacino & 10 & 90 & 3 & 67 & 5 & 80 & 3 & 0 & 10 & 40 & NT & NT & NT & NT \\
\hline Clindamicina & NT & NT & NT & NT & NT & NT & NT & NT & NT & NT & 2 & 0 & 3 & 100 \\
\hline Colistina & 9 & 0 & 3 & 0 & 5 & 0 & 3 & 0 & 8 & 0 & NT & NT & NT & NT \\
\hline Daptomicina & NT & NT & NT & NT & NT & NT & NT & NT & NT & NT & 2 & 0 & 3 & 0 \\
\hline Ertapenem & NT & NT & NT & NT & 5 & 40 & 3 & 0 & 10 & 40 & NT & NT & NT & NT \\
\hline Eritromicina & NT & NT & NT & NT & NT & NT & NT & NT & NT & NT & 2 & 0 & 3 & 100 \\
\hline Gentamicina & 10 & 90 & 3 & 67 & 5 & 80 & 3 & 33 & 10 & 20 & NT & NT & NT & NT \\
\hline Imipinem & 10 & 90 & 3 & 67 & 5 & 0 & 3 & 0 & 10 & 10 & NT & NT & NT & NT \\
\hline Levofloxacino & 10 & 90 & 3 & 67 & 5 & 80 & 3 & 0 & 10 & 40 & NT & NT & NT & NT \\
\hline Linezolide & NT & NT & NT & NT & NT & NT & NT & NT & NT & NT & 2 & 0 & 3 & 67 \\
\hline Meropenem & 10 & 90 & 3 & 67 & 5 & 20 & NT & NT & 10 & 10 & NT & NT & NT & NT \\
\hline Minociclina & NT & NT & NT & NT & NT & NT & NT & NT & NT & NT & 2 & 0 & 3 & 33 \\
\hline Oxacilina & NT & NT & NT & NT & NT & NT & NT & NT & NT & NT & 2 & 0 & 3 & 67 \\
\hline Penicilina & NT & NT & NT & NT & NT & NT & NT & NT & NT & NT & 2 & 100 & 3 & 100 \\
\hline Pipe+tazo & 10 & 90 & 3 & 33 & 5 & 80 & 3 & 33 & NT & NT & NT & NT & NT & NT \\
\hline Rifamicina & NT & NT & NT & NT & NT & NT & NT & NT & NT & NT & 2 & 0 & 3 & 100 \\
\hline Smt+tmp & 10 & 90 & NT & NT & 5 & 80 & 3 & 33 & 10 & 20 & 2 & 0 & 3 & 33 \\
\hline Tigeciclina & NT & NT & NT & NT & 5 & 0 & 3 & 0 & 8 & 0 & 2 & 0 & NT & NT \\
\hline Vancomicina & NT & NT & NT & NT & NT & NT & NT & NT & NT & NT & 2 & 0 & 1 & 0 \\
\hline
\end{tabular}

NT: não testado; n: número de isolados bacterianos; \%: percentual de resistência; pipe+tazo: piperacilina + tazobactam; smt+tmp:sulfametoxazol + trimetoprim; amp+sub: ampicilina + sulbactam; amox+clav: amoxicilina + clavulanato

Quando a infecção é de origem torácica, foram identificadas 27 culturas positivas enquanto 59 foram negativas. Das positivas, 06 corresponderam a hemoculturas, 08 acultura de líquido pleural, 08 de secreção traqueal, 03 de ferida operatória e 02 de uroculturas.

Das bactérias encontradas nesses pacientes, a mais prevalente foi A. baumannii, que correspondeu a $25,9 \%$ dos casos. Quando se analisa o gênero, Staphylococcus pode-se observar a presença em 29,6\% dos casos, divididos entre11,1\% S. aureus, $11,1 \% \mathrm{~S}$. epidermidis e 7,4\% S. haemolyticus. Além disso, 7,4\% de $K$. pneumoniae, 7,4\% de $E$. coli, 7,4\% de S. maltophilia,7,4\% de E. cloacae, 3,7\% de Moraxella catarrhalis, 3,7\% de E.faecium, 3,7\% de E. faecalise 3,7\% de S. cristatus (Figura 3). 


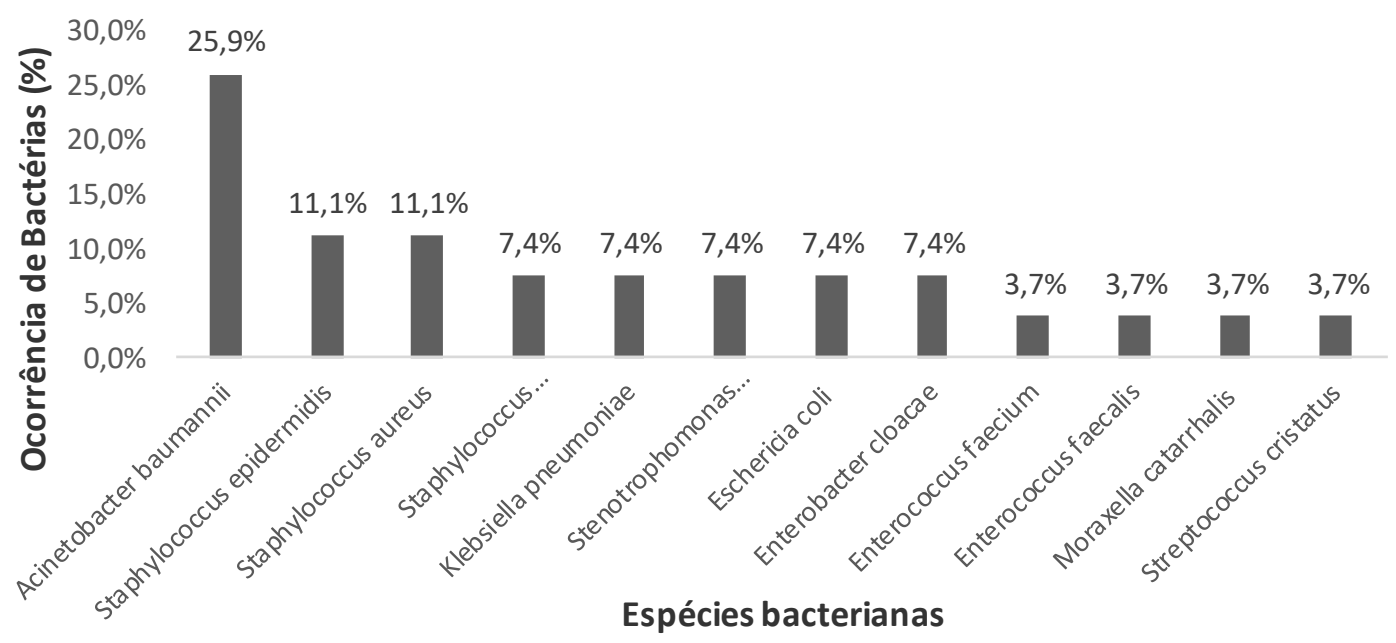

Figura 3 - Ocorrência de bactérias isoladas em culturas coletadas para o diagnóstico de sepse de foco torácico/pulmonar.

Em relação ao A. baumannii, $100 \%$ dos casos testados foram resistentes a ciprofloxacino e levofloxacino, enquanto $100 \%$ foram sensíveis a colistina. Todos os outros antimicrobianos testados a porcentagem de resistência foram superiores a $70 \%$, com a exceção de ampicilina-sulbactam (43\%) e sulfametoxazol-trimetoprim (57\%).

Os isolados em que foram identificados a presença de $S$. aureus, foi observada uma baixa resistência, sendo a maior delas $67 \%$ a penicilina $\mathrm{G}$ e alta sensibilidade para a maioria dos antibióticos, dentre eles $100 \%$ de sensibilidade paras a daptomicina, linezolida, minociclina, rifampicina, sulfametoxazol-trimetoprim, tigeciclina e vancomicina. Já quando se refere a $S$. haemolyticus isso não se repete, uma vez que $100 \%$ dos isolados foram resistentes a penicilina, ampicilina, clindamicina, eritromicina e oxacilina. Padrão este que se assemelha quando se observa o perfil de $S$. epidermidis, com exceção da oxacilina que nesse caso a resistência foi de $67 \%$.

Das culturas com K. pneumoniae os antibiogramas mostraram que dos 17 antimicrobianos testados, 13 apresentaram-se como 50\% resistentes e 50\% sensíveis. Além disso, o único antibiótico com resistência de $100 \%$ foi ampicilina, enquanto que colistina, amicacina e tigeciclina apresentaram 100\% de sensibilidade.

No que se refere a E. coli, $100 \%$ dos isolados foram resistentes a ampicilina, ampicilina-sulbactam, amoxicilina-clavulanato, cefepime, cefazolina, ceftriaxona e cefuroxima. Enquanto $100 \%$ foram sensíveis a amicacina, cefoxitina, colistina, gentamicina, imipenem, meropenem, sulfametoxazol-trimetoprim e piperacilinatazobactam.

\section{Discussão}

No presente estudo, foram analisados 473 pacientes, desses 13,9\% apresentaram infecção comprovada por cultura. Esse índice é alto quando comparado a um trabalho realizado em um Hospital Universitário do Rio Grande do Sul, o qual houve uma baixa prevalência de $\mathrm{IH}(0,9 \%)$ (14). Esse fato deve estar relacionado ao perfil assistencial do hospital do presente trabalho e ao fato do mesmo ser um Hospital conveniado somente ao SUS, referência em uma região que abrange cerca de 2 milhões de habitantes, além de atender demanda espontânea de pacientes vítimas de trauma. Como também ao fato de que como o estudo no qual está sendo comparado foram analisados todos os 
pacientes e não somente aqueles da cirurgia geral.

Um estudo realizado em um Hospital Universitário da cidade de Patos-PB, em 2017, evidenciou que a maioria das pessoas admitidas nas enfermarias da clínica cirúrgica que obtiveram algum tipo de infecção pós-cirúrgica era do sexo masculino (75\%) (15), se equiparando aos dados encontrados no presente trabalho $(72,7 \%)$.

Quando o parâmetro é a idade, o presente estudo teve pacientes com média de 44,3 anos e esta média varia para 39,8 quando se caracteriza como pacientes provenientes da emergência. Já uma pesquisa realizada em um Hospital de Emergência de Uberaba-MG, teve como média de 42,3 anos (16), o que se equipara ao valor encontrado neste estudo.

A maior parte dos pacientes não necessitou de internação em Unidade de Terapia Intensiva (UTI), apenas 19,7\%, o que não é observado na maioria dos estudos, como, por exemplo, no estudo realizado no Hospital Universitário do Rio Grande do Sul, no qual o valor da porcentagem de pacientes com infecção hospitalar internados em UTI foi de $75 \%$ (14). Isso pode ser justificado pela escassez de leitos de terapia intensiva no hospital estudado e pelo perfil dos pacientes estar relacionado a pacientes mais jovens.

Um estudo realizado em João Pessoa, $16 \%$ dos pacientes tiveram infecção de sítio cirúrgico (15), já no presente trabalho, apenas três por cento dos pacientes tiveram esse tipo de complicação pós-operatória.

Em um estudo realizado na Polônia, utilizando o método de vigilância ativa de infecção hospitalar, em pacientes adultos, que passaram mais de 48 horas em uma enfermaria geral de UTI entre 2007 e 2016, os micro-organismos mais identificados foram: A. baumannii (25\%), seguido por Staphylococcus coagulase-negativa (15\%), E. coli $(9 \%)(17)$. O presente estudo não analisou separadamente pacientes em UTI, mas a bactéria com maior incidência também foi $A$. baumannii, além de $E$. coli que corresponderam a 11,9\% cada, seguido de K. pneumoniae $(10,71 \%)$.

Na pesquisa, o achado mais relevante no que se refere ao $A$. baumannii é que o único antimicrobiano com $100 \%$ de sensibilidade em todas as culturas analisadas foi a colistina. Comparando com estudo realizado no Hospital das Clínicas de Recife-UFPE, o Acinetobacter apresentou sensibilidade de $100 \%$ não só a polimixina $\mathrm{B}$, como também a tigeciclina (18). Vale salientar, que muitos hospitais não apresentam padronização do antibiograma nos laudos, não sendo possível analisar a frequência de resistência das bactérias aos antimicrobianos, como mencionado no artigo (14). Fato este que diferencia esta pesquisa com muitos artigos presentes nas páginas de pesquisa virtual.

A. baumannii (genoespécies 2 do complexo ACB) é a mais resistente das genoespécies e tem a maior importância clínica. Esse patógeno é a espécie mais frequentemente isolada (> 90\% dos isolados de Acinetobacter spp.) e está tipicamente associado a surtos no ambiente hospitalar (19).

Em um estudo realizado no Hospital das Clínicas de Recife-UFPE, a análise do perfil de sensibilidade aos antimicrobianos demonstrou que o grupo das Pseudomonas spp. se apresentou sensível a amicacina em 100\% dos casos, seguidos dos antimicrobianos, ciprofloxacino, polimixina B e levofloxacino com $75 \%$ (18).O que se contrapõe a este estudo, visto que essa bactéria foi $100 \%$ sensível somente a colistina, enquanto que $68 \%$ dos casos foram resistentes a todos os outros antibióticos testados no antibiograma, excetuando a piperacilina-tazobactam, que esse número diminui para $33 \%$.

No presente estudo, S. aureus e S. epidermidis se apresentavam como $100 \%$ resistentes a ampicilina, eritromicina e penicilina G. Já em relação a sensibilidade, o $S$. aureus foi sensível a mais bactérias que o S. epidermidis, mas ambos foram $100 \%$ sensíveis a daptomicina e à vancomicina.

No presente estudo, as culturas que foram positivas para E. coli, foram $100 \%$ resistentes à ampicilina-sulbactam, $90 \%$ a ampicilina e $70 \%$ a ceftriaxona, enquanto que 
$100 \%$ foram sensíveis a amicacina, colistina e tigeciclina, esse fato pode estar relacionado a produção de ESBLs do tipo TEM ou SHV, também conhecidas como ceftazidimases, codificadas por plasmídeos $(20,21)$.

Anna Karoeny et al., relataram em seu trabalho que esse mesmo mecanismo - ESBL pode ocorrer também no gênero Klebsiella sp., o que coincide com resultado da presente pesquisa, onde $100 \%$ das $K$. pneumoniae isoladas foram resistentes a ampicilina e cefazolina, $80 \%$ a ampicilina-sulbactam, cefepime, ceftriaxona, ciprofloxacino, gentamicina, levofloxacino, piperacilina-tazobactam e sulfametoxazoltrimetoprim.

Patógenos multirresistentes, como MRSA, VRE, A. baumannii, Enterobacteria ceae produtores de beta-lactamases de espectro estendido e/ou carbapenemases (por exemplo, ESBLs e CREs, respectivamente) e $P$. aeruginosa resistentes ao carbapenêmicos, todos estão sendo isolados com frequência crescente em UTIs. As infecções causadas por esses patógenos resistentes são difíceis de tratar e estão associadas ao aumento da morbidade, mortalidade e custos (22).

\section{Conclusões}

Os resultados encontrados permitem o conhecimento do perfil clínico, epidemiológico e microbiológico dos pacientes admitidos no setor de cirurgia geral, realçando o perfil de sensibilidade e resistência das bactérias encontradas no Hospital Universitário da UNIVASF, localizado em Petrolina-PE.

De acordo com os achados do presente estudo, foi observado que as infecções hospitalares em pacientes do setor de cirurgia geral do Hospital Universitário estão mais relacionadas a infecções abdominais e torácicas, o que diferencia do perfil da maioria dos estudos encontrados.

Baseado nos achados deste estudo sugere-se que estudos futuros sejam realizados, discussões periódicas entre as equipes de saúde deste hospital aconteçam com o objetivo de analisar esses indicadores de taxas de infecção hospitalar, perfil de resistência microbiana, na perspectiva de que essas $\mathrm{IH}$ poderiam ser evitadas, trabalhando junto com $\mathrm{CCIH}$ da instituição, promovendo orientações e atividades de educação continuada, incentivando a participação das equipes a elevaras medidas de controle de infecções, e prover informações para a criação de protocolos de prescrição de antibióticos conforme o perfil de resistência dos micro-organismos e revisão dos mesmos.

\section{Referências}

1. Pereira Júnior GA, Lovato WJ, Carvalho JB, Horta MFV. Abordagem geral trauma abdominal. Revista da Faculdade de Medicina de Ribeirão Preto-USP 2007; 40(4): 518-30.

2. Sanabria A, Valdivieso E, Gomez G, Echeverry G. Prophylactic antibiotics in chest trauma: a metaanalysis of high-quality studies. World J Surg 2006; 30(10): 1843-7.

3. Luchette FA, Barrie PS, Oswanski MF, Spain DA, Mullins CD, Palumbo F, Pasquale MD. Practice Management Guidelines for Prophylactic Antibiotic Use in Tube Thoracostomy for Traumatic Hemopneumothorax: the EAST Practice Management Guidelines Work Group. Eastern Association for Trauma. J Trauma 2000; 48(4): 753-7.

4. Garner JS, Jarvis WR, Emori TG, Horan TC, Hughes JM. CDC definitions for nosocomial infections. Am J Infect Control 1998; 16: 128-40.

5. Allegranzi B, Bagheri Nejad S, Combescure C, Graafmans W Attar H, Donaldson. Burdenofendemi cheal th-care-associated infection in developing countries: systematic review and meta-analysis. The Lancet 2011; (377): 228-24.

6. Radu DM, Jauréguty F, Seguin A, Foulon C, Destable MD, Azorin J, Martinod E. Postoperative 
pneumonia after major pulmonary resections: an unsolved problem in thoracic surgery. Ann Thorac Surg. 2007; 84(5): 1669-73.

7. Rathore MH, Jackson MA. Infection Prevention and Control in Pediatric Ambulatory Settings. Pediatrics 2017; 140(5): 2017-57.

8. Estrela TS. Resistência antimicrobiana: enfoque multilateral e resposta brasileira. Saúde e Política Externa. 20: 1998-2018.

9. Hoecke FV, Deloof NE, Claeys G. Performance evaluation of a modified chromogenic medium, Chrom ID MRSA New, for the detection of methicillin-resistant Staphylococcus aureus from clinical specimens. European Journal of Clinical Microbiology and Infectious Diseases 2011; 30(12) 15951598

10. Quian Q, Venkataraman L, Kirby JE, Gold HS, Yamazumi T. Direct Detection of Methicillin Resistance in Staphylococcus aureus in Blood Culture Broth by Use of a Penicillin Binding Protein 2a Latex Agglutination Test. Journal of Clinical Microbiology 2010; 48(140): 1420-1421.

11. Rosa MB, et al. A Farmácia e o Controle das Infecções Hospitalares. In: GOMES, M J V M. \& REIS, A M M. (Org). Ciências Farmacêuticas: Uma Abordagem em Farmácia Hospitalar 2003; 407-428.

12. Queenan AM, Bush K. Carbapenemases: the Versatile B-Lactamases. Clinical Microbiology 2007; 20: 440-458.

13. Peirano G, Seki LM, Passos VLV, Pinto MCFG, Guerra LR, Asensi MD. Carbapenem-hydrolysing beta-lactamase KPC-2 in Klebsiella pneumoniae isolated in Rio de Janeiro, Brazil. Journal of Antimicrobial Chemotherapy 2009; 63: 265-268.

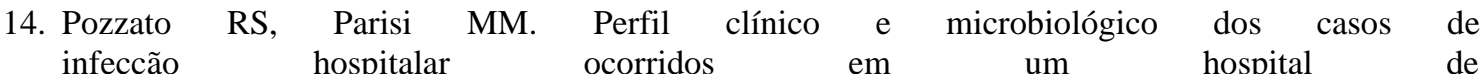
médio porte do noroeste do Rio Grande do Sul. Revista Brasileira de Análises Clínicas 2018;50(3):260-4.

15. Santos ACSL, Leite KNSL, Silva SCR, Lima AKBS, Azevedo SC, Amorim Filho JP. Perfil Epidemiológico de Pacientes com Infecção no Sítio Cirúrgico em um Hospital da Rede Pública. Temas em saúde 2018; 18(2):28-42.

16. Barbosa MH, Luiz RB, Andrade EV, Silva QCG, Mattia AL. Ocorrência De Infecção De Sítio Cirúrgico Em Cirurgias De Urgência E Emergência. Rev. Min. Enferm 2011; 15(2): 254-258.

17. Kołpa M, Wałaszek M, Gniadek A, Wolak Z, Dobroś W Incidence, microbiological profle and risk factors of healthcare-associated infections in intensive care units: a 10 year observation in a provincial hospital in Southern Poland. International Journal of Environmental Research and Public Health 2018; 15 (1): 112.

18. Santos AKS, Araújo JA, de Moura M, Carvalho LRB, Coelho LS, Landim CAP Perfil Microbiológico Das Infecções Hospitalares Nas Unidades de Terapia Intensiva. Revista de enfermagem - UFPE 2016; 10 (3): 1432-40.

19. Chuang YC, Sheng WH, Li SY, Lin YC, Wang JT, Chen YC, Chang SC. Influence of genospecies of Acinetobacter baumannii complex on clinical outcomes of patients with Acinetobacter bacteremia. Clin Infect Dis 2011; 52(3): 352-60.

20. Neves PR, Mamizuka EM, Levy CE, Lincopan N Pseudomonas aeruginosa multirresistente: um problema endêmico no Brasil. J Bras Patol Med Lab 2011; 47(4): 409-420.

21. Bortoli, AAMD. Perfil de sensibilidade bacteriana em uroculturas de pacientes internados na enfermaria geriátrica do hospital do servidor público municipal. 2011. 24p.tese (residência médica). São Paulo.

22. Hidron AI, Edwards JR, Patel J, Horan TC, Sievert DM, Pollock DA, Fridkin SK; National Healthcare Safety Network Team; Participating National Healthcare Safety Network Facilities.. NHSN annual update: antimicrobial-resistant pathogens associated with healthcare-associated infections: annual summary of data reported to the National Healthcare Safety Network at the Centers for Disease Control and Prevention, 2006-2007. Infect Control Hosp Epidemiol. 2008; 29(11): 996-1011. 